九州大学学術情報リポジトリ

Kyushu University Institutional Repository

Variable and boundary selection for functional data via multiclass logistic regression modeling

Matsui, Hidetoshi

Faculty of Mathematics, Kyushu University

http://hdl. hand le. net/2324/26121

出版情報 : Computational Statistics \& Data Analysis. 78, pp.176-185, 2014-10. Elsevier バージョン：

権利関係 : 


\section{Preprint Series}

Kyushu University

The Global COE Program

Math-for-Industry Education \& Research Hub

\section{Variable and boundary selection for functional data via multiclass logistic regression modeling}

\section{Hidetoshi Matsui}

MI 2013-7

( Received March 14, 2013 )

Faculty of Mathematics

Kyushu University

Fukuoka, JAPAN 


\title{
Variable and boundary selection for functional data via multiclass logistic regression modeling
}

\author{
Hidetoshi Matsui \\ Faculty of Mathematics, Kyushu University \\ 744 Motooka, Nishi-ku, Fukuoka 819-0395, Japan. \\ hmatsui@math.kyushu-u.ac.jp
}

\begin{abstract}
: $\ell_{1}$ penalties such as the lasso provide solutions with some coefficients to be exactly zeros, which lead to variable selection in regression settings. They also can select variables which affect the classification by being applied to the logistic regression model. We focus on the form of $\ell_{1}$ penalties in logistic regression models for functional data, especially in the case for classifying the functions into three or more groups. We provide penalties that appropriately select variables in the functional multinomial regression modeling. Simulation and real data analysis show that we should select the form of the penalty in accordance with the purpose of the analysis.
\end{abstract}

Key Words and Phrases: Functional Data Analysis, Lasso, Logistic regression model, Model selection, Regularization.

\section{Introduction}

Variable selection is one of crucial issues in regression analysis. The lasso by Tibshirani (1996) and its offshoots provide us an unified approach for estimation and variable selection problems, and therefore they are broadly applied in several fields (see, e.g. Hastie et al., 2009). We can also select variables which affect to classification problems by applying these types of penalties to logistic regression models (Krishnapuram et al., 2005; Park and Hastie, 2007; Friedman et al., 2010). The logistic regression model is one of the most useful tools for classifying data by providing posterior probabilities which group the data belong to.

In this paper we consider the cases for classifying data into three or more groups using the multiclass logistic regression model, which contains multiple parameters in each variable, as well as a multivariate linear model. Several works have been provided for multivariate linear or multiclass logistic regression models. Turlach et al. (2005) proposed a new penalty to estimate multivariate linear models. They imposed an $\ell_{1}$ sum of maximum absolute values $\left(\ell_{\infty}\right.$ norm) of coefficients with respect to multiple responses, and also generalized it to the $\ell_{1}$ sum of $\ell_{q}(q \geq 1)$ penalties. Then afterwards Yuan et al. (2007)and Obozinski et al. (2011) let the penalty denote $\ell_{1} / \ell_{q}$ and investigated theoretical properties of it. Furthermore, Obozinski et al. (2010) proposed a new algorithm for estimating the multinomial logistic regression model with the $\ell_{1} / \ell_{q}$ regularization for $q=1,2$. 
When the data to be classified are repeatedly measured over time, it is natural that they are represented by some functional forms. Ramsay and Silverman (2005) established this type of analysis and call it the Functional data analysis (FDA). FDA is one of the most useful methods for effectively analyzing such discretely observed data and has received considerable attentions in various fields (Ramsay and Silverman, 2002; Ferraty and Vieu, 2006; Ferraty, 2011). The basic idea behind FDA is to express repeated measurement data as a smooth function and then draw information from the collection of functional data. FDA includes extensions of traditional analyses such as principal component, discriminant and regression analysis (James et al., 2000; James, 2002).

In this paper we consider the variable selection problem for classifying functional data using the logistic regression model via the sparse regularization. Repeated measurement data are represented by basis expansions, and then the functional logistic regression model is estimated by the penalized maximum likelihood method with the help of sparsity penalties. We propose a new penalty, denoted by $\ell_{1} \ell_{2} / \ell_{q}$ with $q=1,2$ here, for appropriately estimating and selecting variables or boundaries for the multiclass functional logistic regression model. Furthermore, the estimated model is evaluated by the model selection criterion since the model evaluation problem is a crucial issue. We examine the proposed method through Monte Carlo simulations and real data examples.

This paper is organized as follows. Section 2 provides a multiclass logistic regression model for functional data. Section 3 shows the method for estimating and evaluating the model. We apply the proposed method to the analysis of simulated data and real data in Section 4 and Section 5 respectively. Concluding remarks are given in Section 6 .

\section{Multinomial logistic regression model for functional data}

Suppose that we have $n$ sets of functional data and a class label $\left\{\left(\boldsymbol{x}_{\alpha}(t), g_{\alpha}\right) ; \alpha=1, \ldots, n\right\}$, where $\boldsymbol{x}_{\alpha}(t)=\left(x_{\alpha 1}(t), \ldots, x_{\alpha p}(t)\right)^{T}$ are predictors given as functions and $g_{\alpha} \in\{1, \ldots, L\}$ are classes which $\boldsymbol{x}_{\alpha}$ belongs to. In the classification setting, we apply the Bayes rule which assigns $\boldsymbol{x}_{\alpha}$ to class $g_{\alpha}=l$ with the maximum posterior probability given $\boldsymbol{x}_{\alpha}$, denoted by $\operatorname{Pr}\left(g_{\alpha}=l \mid \boldsymbol{x}_{\alpha}\right)$. Then the logistic regression model is given by the log-odds of posterior probabilities:

$$
\log \left\{\frac{\operatorname{Pr}\left(g_{\alpha}=l \mid \boldsymbol{x}_{\alpha}\right)}{\operatorname{Pr}\left(g_{\alpha}=L \mid \boldsymbol{x}_{\alpha}\right)}\right\}=\beta_{l 0}+\sum_{j=1}^{p} \int x_{\alpha j}(t) \beta_{l j}(t) d t,
$$

where $\beta_{l 0}$ is an intercept and $\beta_{l j}(t)$ are coefficient functions. We consider that $x_{\alpha j}(t)$ is expressed by basis expansions by

$$
x_{\alpha j}(t)=\sum_{m=1}^{M_{j}} w_{\alpha j m} \phi_{j m}(t)=\boldsymbol{w}_{\alpha j}^{T} \boldsymbol{\phi}_{j}(t),
$$


where $\phi_{j}(t)=\left(\phi_{j 1}(t), \ldots, \phi_{j M_{j}}(t)\right)^{T}$ are vectors of basis functions such as $B$-splines or radial basis functions, $\boldsymbol{w}_{\alpha j}=\left(w_{\alpha j 1}, \ldots, w_{\alpha j M_{j}}\right)$ are coefficient vectors. Since the data are originally observed at discrete time points, we obtain functional data $x_{\alpha j}(t)$ using a smoothing method with basis expansions in advance. In other words, $w_{\alpha j}$ are obtained before constructing the functional logistic regression model (1). Details of the smoothing method are described in Araki et al. (2009b). Furthermore, $\beta_{l j}(t)$ are also expressed by basis expansions

$$
\beta_{l j}(t)=\sum_{m=1}^{M_{j}} b_{l j m} \phi_{j m}(t)=\boldsymbol{b}_{l j}^{T} \boldsymbol{\phi}_{j}(t)
$$

where $\boldsymbol{b}_{l j}=\left(b_{l j 1}, \ldots, b_{l j M_{j}}\right)^{T}$ are vectors of coefficient parameters.

Using a notation $\pi_{l}\left(\boldsymbol{x}_{\alpha} ; \boldsymbol{b}\right)=\operatorname{Pr}\left(g_{\alpha}=l \mid \boldsymbol{x}_{\alpha}\right)$ with $\boldsymbol{b}=\left(\boldsymbol{b}_{1}^{T}, \ldots, \boldsymbol{b}_{(L-1)}^{T}\right)^{T}$ and $\boldsymbol{b}_{l}=$ $\left(\beta_{l 0}, \boldsymbol{b}_{l 1}^{T}, \ldots, \boldsymbol{b}_{l p}^{T}\right)^{T}$ since it is controlled by $\boldsymbol{b}$, we can re-express the functional logistic regression model (1) as

$$
\log \left\{\frac{\pi_{l}\left(\boldsymbol{x}_{\alpha} ; \boldsymbol{b}\right)}{\pi_{L}\left(\boldsymbol{x}_{\alpha} ; \boldsymbol{b}\right)}\right\}=\beta_{l 0}+\sum_{j=1}^{p} \boldsymbol{w}_{\alpha j}^{T} \Phi_{j} \boldsymbol{b}_{l j}=\boldsymbol{z}_{\alpha}^{T} \boldsymbol{b}_{l},
$$

where $\boldsymbol{z}_{\alpha}=\left(1, \boldsymbol{w}_{\alpha 1}^{T} \Phi_{1}, \ldots, \boldsymbol{w}_{\alpha p}^{T} \Phi_{p}\right)$ and $\Phi_{j}=\int \boldsymbol{\phi}_{j}^{T}(t) \boldsymbol{\phi}_{j}(t)$. It follows from (1) that the posterior probability is given by

$$
\begin{aligned}
\pi_{l}\left(\boldsymbol{x}_{\alpha} ; \boldsymbol{b}\right) & =\frac{\exp \left(\boldsymbol{z}_{\alpha}^{T} \boldsymbol{b}_{l}\right)}{1+\sum_{h=1}^{L-1} \exp \left(\boldsymbol{z}_{\alpha}^{T} \boldsymbol{b}_{h}\right)} \quad(l=1, \ldots, L-1), \\
\pi_{L}\left(\boldsymbol{x}_{\alpha} ; \boldsymbol{b}\right) & =\frac{1}{1+\sum_{h=1}^{L-1} \exp \left(\boldsymbol{z}_{\alpha}^{T} \boldsymbol{b}_{h}\right)} .
\end{aligned}
$$

We define vectors of response variables $\boldsymbol{y}_{\alpha}$ which indicate class labels as

$$
\boldsymbol{y}_{\alpha}=\left(y_{\alpha 1}, \ldots, y_{\alpha(L-1)}\right)^{T}= \begin{cases}(0, \ldots, 0, \stackrel{(l)}{1}, 0, \ldots, 0)^{T} & \text { if } g_{\alpha}=l, \quad l=1, \ldots, L-1 \\ (0, \ldots, 0)^{T} & \text { if } g_{\alpha}=L\end{cases}
$$

Then the functional logistic regression model has the following probability function

$$
f\left(\boldsymbol{y}_{\alpha} \mid \boldsymbol{x}_{\alpha} ; \boldsymbol{b}\right)=\prod_{l=1}^{L-1} \pi_{l}\left(\boldsymbol{x}_{\alpha} ; \boldsymbol{b}\right)^{y_{\alpha l}} \pi_{L}\left(\boldsymbol{x}_{\alpha} ; \boldsymbol{b}\right)^{1-\sum_{h=1}^{L-1} y_{\alpha h}}
$$

\section{Estimation by the sparse regularization}

In order to construct the statistical model, we estimate the functional logistic model (1) by the penalized likelihood method, which maximizes the penalized log-likelihood function given in the form of

$$
l_{\lambda}(\boldsymbol{b})=l(\boldsymbol{b})-n \lambda P(\boldsymbol{b})
$$


where $l(\boldsymbol{b})=\sum_{\alpha=1}^{n} \log f\left(\boldsymbol{y}_{\alpha} \mid \boldsymbol{x}_{\alpha} ; \boldsymbol{b}\right)$ is a log-likelihood function, $\lambda$ is a regularization parameter which controls the effect of the penalty and $P(\cdot)$ is a penalty function. We can also select variables which actually have an influence on the classification by employing one of the $\ell_{1}$ type penalties on $P(\cdot)$. Here we adopt the lasso as the $\ell_{1}$ type penalty in this paper. Now we consider two types of penalties for $P(\cdot)$ :

$$
P_{(1)}(\boldsymbol{b})=\sqrt{M_{j}} \sum_{j=1}^{p} \sum_{l=1}^{L-1}\left\|\boldsymbol{b}_{l j}\right\|_{2}, \quad P_{(2)}(\boldsymbol{b})=\sqrt{M_{j}(L-1)} \sum_{j=1}^{p}\left\{\sum_{l=1}^{L-1}\left\|\boldsymbol{b}_{l j}\right\|_{2}^{2}\right\}^{\frac{1}{2}} .
$$

In order to select variables appropriately we need to treat $w_{\alpha j 1}, \ldots, w_{\alpha j M_{j}}$ as grouped variables and thus apply the group lasso (Yuan and Lin, 2006) to the corresponding coefficients $b_{l j 1}, \ldots, b_{l j M_{j}}$, otherwise we fail to select functional variables (Matsui and Konishi, 2011). In addition, $P_{(1)}$ imposes $\ell_{1}$ norms of coefficient parameters for each class. On the other hand, $P_{(2)}$ treats $L-1$ parameters as grouped parameters gain. The former penalty shrinks each coefficient towards exactly zero, whereas the latter shrinks all the $L-1$ parameter vectors in the $j$-th variable towards zero simultaneously. We denote $P_{(1)}$ and $P_{(2)}$ as $\ell_{1} \ell_{2} / \ell_{1}$ and $\ell_{1} \ell_{2} / \ell_{2}$ penalties respectively. If we consider the case $M_{j}=1$ for all $j$, they correspond to $\ell_{1} / \ell_{q}$ penalties by Obozinski et al. $(2010,2011)$ with $q=1,2$ respectively, and therefore they are regarded as natural extensions of the $\ell_{1} / \ell_{q}$ penalties. We can also express the $\ell_{1} \ell_{2} / \ell_{q}$ norm in the following form.

$$
P_{(q)}(\boldsymbol{b})=C \sum_{j=1}^{p}\left\{\sum_{l=1}^{L-1}\left\|\boldsymbol{b}_{l j}\right\|_{2}^{q}\right\}^{\frac{1}{q}}
$$

here we denoted a constant independent of $\boldsymbol{b}$ as $C$.

Since the penalized log-likelihood function involves the $\ell_{1}$ norm of coefficients it is difficult to derive estimates analytically. We apply the local quadratic approximation to the penalty (Tibshirani, 1996; Fan and Li, 2001). Then parameters are updated in the following form:

$$
\boldsymbol{b}^{(k+1)}=\boldsymbol{b}^{(k)}-\left\{\left.\frac{\partial^{2} l(\boldsymbol{b})}{\partial \boldsymbol{b} \partial \boldsymbol{b}^{T}}\right|_{b^{(k)}}-n \Sigma\left(\boldsymbol{b}^{(k)}\right)\right\}^{-1}\left\{\left.\frac{\partial l(\boldsymbol{b})}{\partial \boldsymbol{b}}\right|_{b^{(k)}}-n \Sigma\left(\boldsymbol{b}^{(k)}\right) \boldsymbol{b}^{(k)}\right\},
$$

where $\Sigma(\boldsymbol{b})$ is given by

$$
\begin{aligned}
& \Sigma(\boldsymbol{b})=\operatorname{diag}\left\{\Sigma\left(\boldsymbol{b}_{1}\right), \ldots, \Sigma\left(\boldsymbol{b}_{(L-1)}\right)\right\}, \\
& \Sigma\left(\boldsymbol{b}_{l}\right)=\left\{\begin{array}{ll}
\operatorname{diag}\left\{\frac{P_{(1)}^{\prime}\left(\left|b_{l 1}\right|\right)}{\left|b_{l 1}\right|}, \ldots, \frac{P_{(1)}^{\prime}\left(\left|b_{l p}\right|\right)}{\left|b_{l p}\right|}\right\} & \text { for } P_{(1)}, \\
\operatorname{diag}\left\{\frac{P_{(2)}^{\prime}\left(\left\|\boldsymbol{b}_{(1)}\right\|\right)}{\left\|\boldsymbol{b}_{(1)}\right\|}, \ldots, \frac{P_{(2)}^{\prime}\left(\left\|\boldsymbol{b}_{(p)}\right\|\right)}{\left\|\boldsymbol{b}_{(p)}\right\|}\right\} & \text { for } P_{(2)}
\end{array} \quad\left(\boldsymbol{b}_{(j)}=\left(b_{1 j}, \ldots, b_{(L-1) j}\right)^{T}\right) .\right.
\end{aligned}
$$

This update is continued until convergence, and then we obtain a estimated coefficient vector $\hat{\boldsymbol{b}}$. 
Statistical models estimated by the penalized likelihood method depend on regularization parameters and it is a crucial issue to select appropriate values of them. In this case we need to select an appropriate value of $\lambda$ in the log-likelihood function (5). We use a model selection criterion BIC originally proposed by Schwarz (1978). For the sparse regularization problem, Wang et al. (2007) proved that the BIC select the true model consistently for the SCAD regularization setting. The BIC for evaluating models estimated by the penalized maximum likelihood method with the $\ell_{1}$ penalty is given by

$$
\begin{aligned}
\mathrm{BIC} & =-2 l(\hat{\boldsymbol{b}})+\widetilde{d f} \log n \\
& =\sum_{\alpha=1}^{n} \sum_{l=1}^{L-1}\left\{y_{\alpha l} \log \pi_{l}\left(\boldsymbol{z}_{\alpha} ; \hat{\boldsymbol{b}}\right)+\left(1-\sum_{h=1}^{L-1} y_{\alpha h}\right) \log \pi_{L}\left(\boldsymbol{z}_{\alpha} ; \hat{\boldsymbol{b}}\right)\right\}+\widetilde{d f} \log n,
\end{aligned}
$$

where $\widetilde{d f}$ is an effective degrees of freedom. Although Zou et al. (2007) derived a degrees of freedom for the model estimated by the lasso-type regularization, it is not given in the logistic settings. Konishi et al. (2004) proposed a model selection criterion GBIC for evaluating models estimated by the regularization method, though, it needs a second derivative of the penalized likelihood function. On the other hand, they also derived AIC or BIC type criterion with effective degrees of freedoms in the logistic regression model. Using this idea, the degrees of freedom here is given by

$$
\widetilde{d f}=\operatorname{tr}\left\{W \tilde{Z}_{\mathcal{A}}\left(\tilde{Z}_{\mathcal{A}}^{T} W \tilde{Z}_{\mathcal{A}}+n \Sigma\left(\hat{\boldsymbol{b}}_{\mathcal{A}}\right)\right)^{-1} \tilde{Z}_{\mathcal{A}}^{T}\right\},
$$

where

$$
\begin{aligned}
\tilde{Z}= & I_{L-1} \otimes Z, \quad Z=\left(\boldsymbol{z}_{1}^{T}, \ldots, \boldsymbol{z}_{n}^{T}\right), \\
W^{(k)}= & \left(W_{h l}^{(k)}\right)_{h l}, \\
W_{h l}^{(k)}= & \left\{\begin{array}{c}
\operatorname{diag}\left\{\pi_{l}\left(\boldsymbol{z}_{1} ; \hat{\boldsymbol{b}}\right)\left(1-\pi_{l}\left(\boldsymbol{z}_{1} ; \hat{\boldsymbol{b}}\right)\right),\right. \\
\left.\ldots, \pi_{l}\left(\boldsymbol{z}_{n} ; \hat{\boldsymbol{b}}\right)\left(1-\pi_{l}\left(\boldsymbol{z}_{n} ; \hat{\boldsymbol{b}}\right)\right)\right\} \quad(h=l), \\
\operatorname{diag}\left\{-\pi_{h}\left(\boldsymbol{z}_{1} ; \hat{\boldsymbol{b}}\right) \pi_{l}\left(\boldsymbol{z}_{1} ; \hat{\boldsymbol{b}}\right),\right. \\
\left.\ldots,-\pi_{h}\left(\boldsymbol{z}_{n} ; \hat{\boldsymbol{b}}\right) \pi_{l}\left(\boldsymbol{z}_{n} ; \hat{\boldsymbol{b}}\right)\right\} \quad(h \neq l)
\end{array}\right.
\end{aligned}
$$

and the suffix $\mathcal{A}$ denotes an active set of $\boldsymbol{b}$. We select the $\lambda$ which minimizes the BIC and then adopt the corresponding model as an optimal model.

\section{Simulation}

In order to investigate the effectiveness and the behavior of the proposed method, we conducted Monte Carlo simulations. We simulated multiple predictors, essentially given as functions having three classes. In this example we aim to examine whether the proposed method appropriately select variables which affect to the classification. 
First, we generated $2 n$ sets of $p$ predictors each of which were repeatedly measured at several time points; $\left\{\left(x_{\alpha i 1}, \ldots, x_{\alpha i p}\right) ; \alpha=1, \ldots, 2 n, i=1, \ldots, n_{i}\right\}$, where $x_{\alpha i j}(j=$ $1, \ldots, p)$ are assumed to be obtained from $x_{\alpha i j}=u_{\alpha j}\left(t_{i}\right)+\varepsilon_{\alpha i j}$ with uniformly distributed $n_{i}$ observational points $t_{i}$ and $\varepsilon_{\alpha i j} \sim N\left(0,\left(\sigma R_{\alpha j}\right)^{2}\right), R_{\alpha j}=\max _{i}\left(u_{\alpha j}\left(t_{i}\right)\right)-\min _{i}\left(u_{\alpha j}\left(t_{i}\right)\right)$. We divided the data with sample size $2 n$ in half in order to use them as training and test data. Here we consider a three-class classification problem, and therefore we trisected the data and then assigned each class with them. We set true functions $u_{\alpha j}(t)$ as follows:

$$
\begin{array}{ll}
u_{\alpha 1}(t)=t^{3}+a_{1} t^{2}+a_{2} t+a_{3}, & u_{\alpha 2}(t)=b_{1} \sin \left(b_{2} \pi\left(t-b_{3}\right)\right), \\
u_{\alpha 3}(t)=c_{1} \exp \left(c_{2} t\right)\left(t-c_{3}\right)^{2}, & u_{\alpha 4}(t)=d_{1} \sin (\pi t)+d_{2},
\end{array} \quad t \in[-1,1],
$$

where they are controlled by following random numbers for classes $g=1,2,3$ respectively.

$$
\begin{array}{ccccc} 
& \text { Class } 1 & \text { Class } 2 & \text { Class } 3 \\
a_{1} & \sim N\left(3.0,0.1^{2}\right), & N\left(2.0,0.1^{2}\right), & N\left(4.0,0.1^{2}\right), \\
u_{1}: & a_{2} \sim N\left(0.5,0.2^{2}\right), & N\left(0.3,0.2^{2}\right), & N\left(0,0.2^{2}\right), \\
a_{3} \sim N\left(1.0,0.5^{2}\right), & N\left(1.0,0.5^{2}\right), & N\left(2.0,0.5^{2}\right), \\
b_{1} \sim U(0.4,0.7), & U(0.8,1.1), & U(0.4,0.7), \\
u_{2}: & b_{2} \sim U(0.9,1.2), & U(0.4,0.7), & U(0.9,0.7), \\
b_{3} \sim N\left(0.2,0.1^{2}\right), & N\left(0.5,0.1^{2}\right), & N\left(0.2,0.7^{2}\right), \\
c_{1} & \sim U(0.7,1.1), & U(0.6,0.9), & U(0.6,0.9), \\
u_{3}: & c_{2} \sim U(0.7,1.2), & \sim(0.8,1.4), & U(0.8,1.4), \\
& c_{3} \sim N(0.8,1.4), & N\left(0.5,0.1^{2}\right), & N\left(0.5,0.1^{2}\right), \\
u_{4}: & d_{1} \sim U(0.5,3.0), & U(0.5,3.0), & U(0.5,3.0), \\
& d_{2} \sim N(0.2,0.5), & N(0.2,0.5), & N(0.2,0.5) .
\end{array}
$$

Note that there are no classification boundaries for Class 1 and 3 on $u_{2}$, Class 2 and 3 on $u_{3}$ and all classes on $u_{4}$, as seen in Table 1 since they respectively have same settings for the random number generation. Especially $u_{4}$ does not affect the classification. These facts show that the coefficient functions in the model (1) should be $\beta_{12}(t)=0, \beta_{23}(t)=0$ and $\beta_{14}(t)=\beta_{24}(t)=0$.

As the first step of the analysis, we converted longitudinal predictors $x_{\alpha i j}$ into functions. Since they contain additive noises we applied the smoothing method with basis expansions, then obtained functional data sets $x_{\alpha j}(t)$. We used radial basis functions with the idea of $B$-splines by Kawano and Konishi (2007) for basis functions $\boldsymbol{\phi}_{j}(t)$ in $(2)$ and (3). In order to reduce computational burden, numbers of basis functions are supposed to be 6 for all variables. Then after constructing the functional logistic regression model, we estimated it by the maximum penalized likelihood method with penalties $P_{(1)}$ and $P_{(2)}$ in (6) and evaluated the model by BIC. We repeated them 100 times for $n=75,150,300$ and $\sigma=0.05,0.2$ and then investigated about results of errors and variable selections for two penalties.

Table 1 shows numbers selected by the above method with penalties $P_{(1)}$ and $P_{(2)}$ for 100 repetition, where " $\beta_{1 j} \beta_{2 j}$ " in the table denotes numbers which both $\beta_{1 j}$ and $\beta_{2 j}$ 

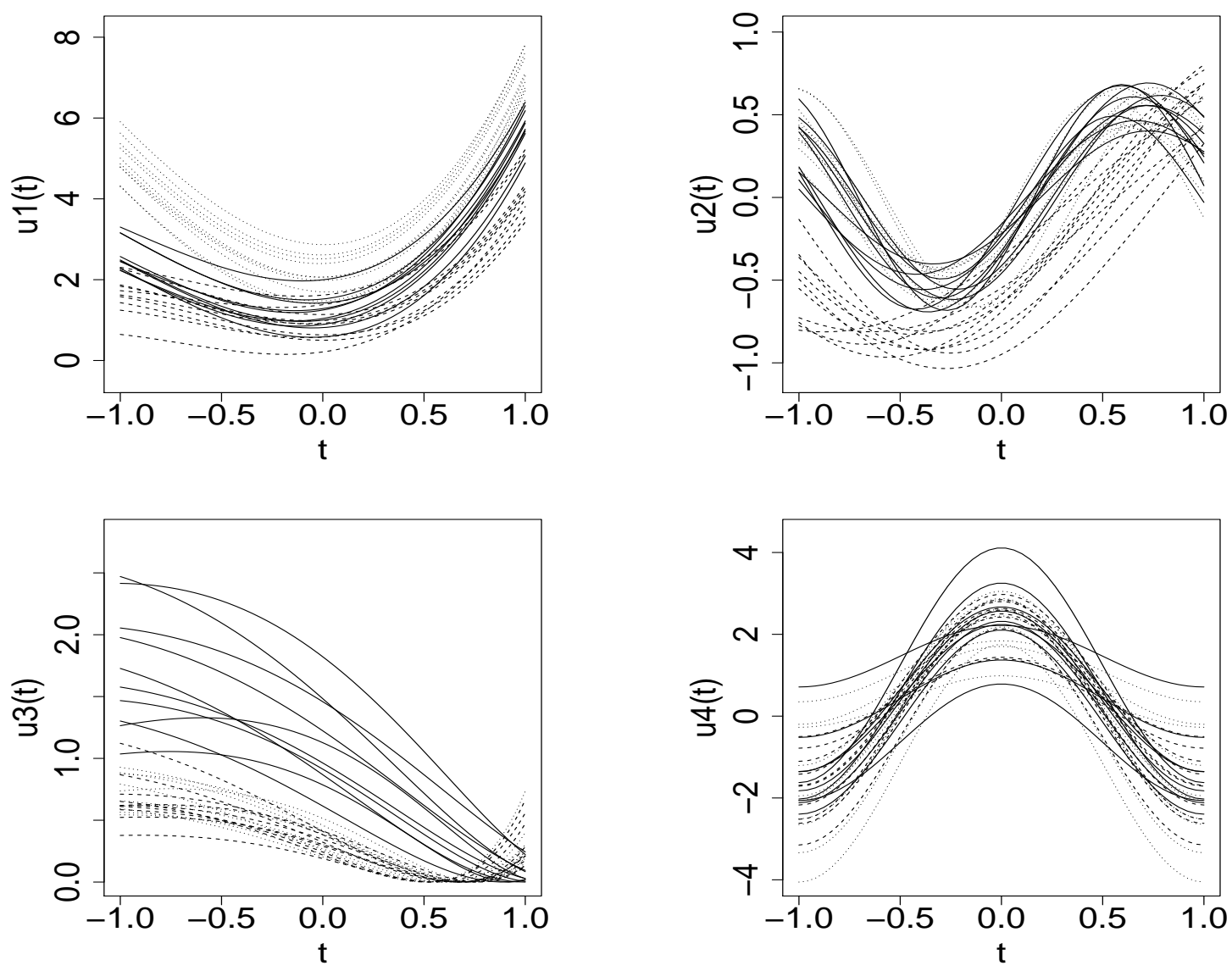

Figure 1: True functions for $u_{j}(j=1, \ldots, 4$ from top left to bottom right $)$ used in the simulation study. Solid, dashed and dotted lines represent functions for Class 1, 2 and 3 respectively.

are estimated to be non-zeros, " $\beta_{l j}$ " for $l=1,2$ denotes numbers which either $\beta_{1 j}$ or $\beta_{2 j}$ is estimated to be non-zero respectively and " $\phi$ " is numbers which both coefficients are estimated to be zeros. We can find that the penalty $P_{(1)}$ shrinks some of coefficient functions to be zeros appropriately, especially for variable 1 and 3 . Thus it selects which classification boundary is important or not for each variable. On the other hand, $P_{(2)}$ selects all or no coefficients for each variable owing to the form of the penalty and hence it cannot select boundaries individually like $P_{(1)}$. However, it excludes variable 4 completely more than $P_{(1)}$, which indicates that $P_{(2)}$ is more suitable for the purpose of only excluding variables themselves which affect the classification. We also obtained training and test errors and selected values of regularization parameters in Table 2. It indicates that the penalty $P_{(1)}$ gives smaller test errors than $P_{(2)}$ in all cases.

Note that we did not mention a classification boundary between Class 1 and 2. We can directly derive the coefficients for the boundary as $\hat{\beta}_{1 j}-\hat{\beta}_{2 j}$, and if there are no boundaries for Class 1 and 2 there should be $\hat{\beta}_{1 j}=\hat{\beta}_{2 j}$. However, the above method does not estimate like it and therefore we cannot estimate appropriately, which reminds to be 

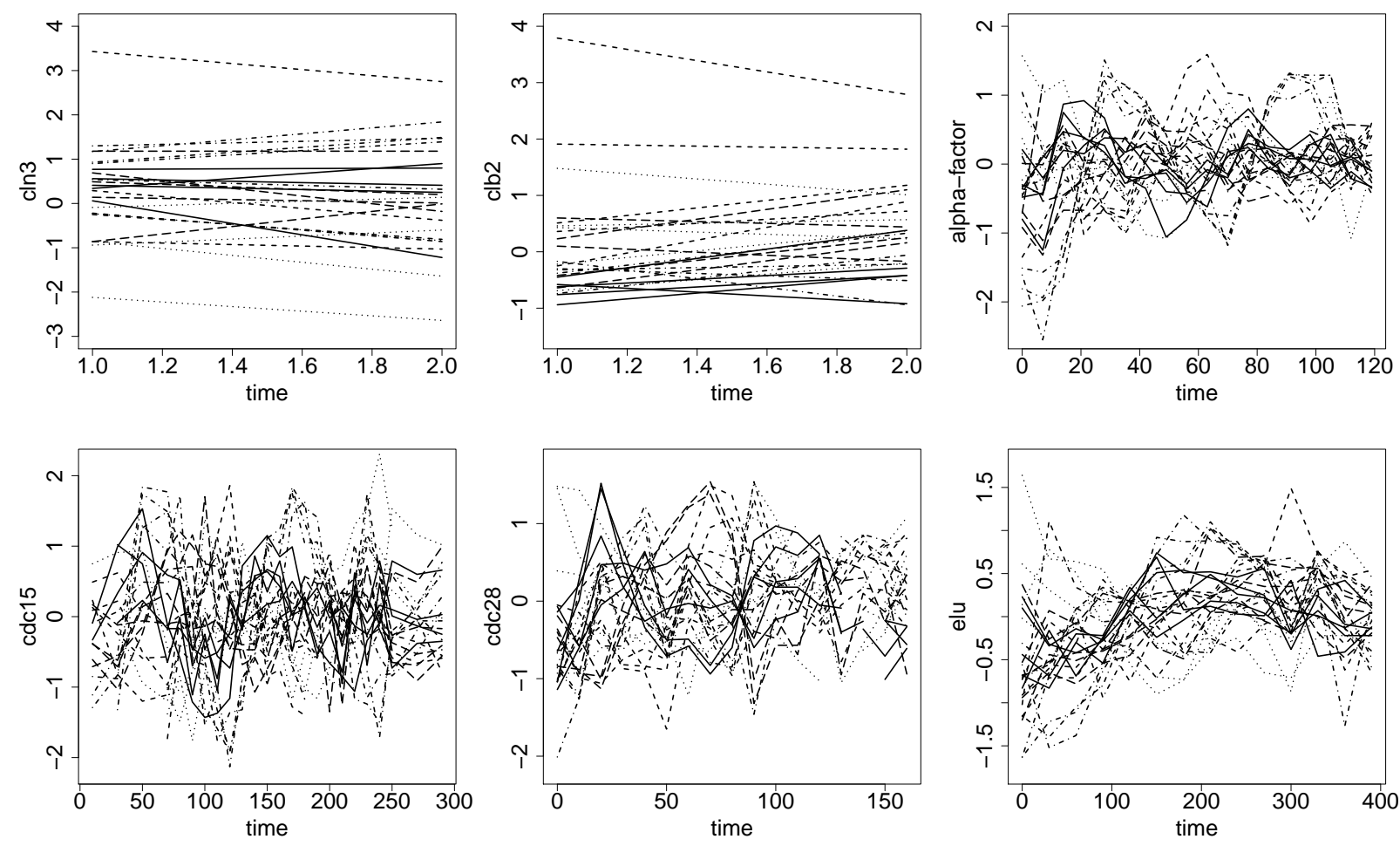

Figure 2: Yeast cell cycle gene expression profiles for each synchronization. Each plot consists of 5 genes from 5 classes. G1 (solid), G2/M (dashed), M/G1 (dotted), S (dotdashed), S/G2 (long dashed).

a future work.

\section{Real data example}

We applied the functional logistic regression modeling to the analysis of yeast cell cycle gene expression data. Spellman et al. (1998) measured expression profiles for 6,178 genome-wide genes in the yeast genome using cDNA microarrays over about two cell cycles. The data contain 77 microarrays consisting of several types of time course synchronization; "cln3" (2 points), "clb2" (2 points), " $\alpha$-factor" (18 points), "cdc15" (24 points), "cdc28" (17 points) and "elu" (14 points). Spellman et al. (1998) classified 800 genes into 5 groups, G1, G2/M, M/G1, S and S/G2, by the clustering method from the above 77 experiments. Figure 2 shows examples for each synchronization. Araki et al. (2009a) classified genes by using the "cdc15" experiments as functional data and examined the misclassified data from the posterior probabilities. Here we aim to confirm that these experiments actually affect the classification.

Since there are some missing values in expression profiles we excluded some genes from data by following two rules: (1) Genes with at least one missing value for "cln3" and "clb2" respectively are excluded. (2) Those with more than 10 missing values in all for 
" $\alpha$-factor", "cdc15", "cdc28" and "elu" are also excluded. Although the number of genes with no missing values are only 72 , we can easily apply the regression model even if there are some (not excessively many) missing values by converting them into functional data. The resulting 657 genes are used for this analysis. First we converted time-course data except for "cln3" and "clb2" into functions. They are expressed by basis expansions with 4 basis functions, which was selected in the functionalization step. Remaining variables "cln3" and "clb2", each of which have only 2 time points, were treated as vector data rather than functional data, and then we treated variables corresponding to 2 time points for each variable as grouped variables. Next we constructed a functional logistic model

$$
\log \left\{\frac{\operatorname{Pr}\left(g_{\alpha}=l \mid \boldsymbol{x}_{\alpha}\right)}{\operatorname{Pr}\left(g_{\alpha}=L \mid \boldsymbol{x}_{\alpha}\right)}\right\}=\beta_{l 0}+\sum_{j=1}^{2} \sum_{j^{\prime}=1}^{2} x_{\alpha j_{j^{\prime}}} \beta_{l j_{j^{\prime}}}+\sum_{j=3}^{6} \int x_{\alpha j}(t) \beta_{l j}(t) d t,
$$

which is a special case of $(1)$, where $X_{j}(j=1, \ldots, 6)$ respectively correspond to "cln3", "clb2", " $\alpha$-factor", "cdc15", "cdc28" and "elu". The model was estimated by the penalized likelihood method and evaluated by BIC. Furthermore we altered the class label $L$ in the left hand side of (7) and repeatedly estimated in order to investigate all coefficients for classification boundaries. We repeated this process for 100 bootstrap samples, and then investigated which variable or boundary affects the classification.

Table 3 shows the numbers of selected variables in 100 bootstrap samples for the penalty $P_{(1)}$. We can find that most coefficients are estimated to be non-zeros. However, the coefficient for the boundary between M/G1 and S/G2 is hardly selected for "clb2", and similarly that between $\mathrm{M} / \mathrm{G} 1$ and $\mathrm{S}$ is often excluded. It reveals that the variable "clb2" does not affect the classification. On the other hand, the penalty $P_{(2)}$ selected all variables for 100 repetitions. It indicates the variables themselves are actually relevant to the classification.

\section{Concluding remarks}

We have proposed a form of penalty for constructing functional multinomial logistic regression models. We derived the estimation and evaluation procedures for the model with the $\ell_{1} \ell_{2} / \ell_{q}$ penalty for $q=1,2$. The model was fitted by the penalized maximum likelihood method, and the regularization parameter involved in the model was selected by the model selection criterion. Monte Carlo simulations were conducted in order to investigate the effects for the accuracy of prediction and variable selection. Results show that $\ell_{1} \ell_{2} / \ell_{1}$ and $\ell_{1} \ell_{2} / \ell_{2}$ penalties give different result and therefore we should select these penalties for different uses.

As described in the end of Section 4, there are cases that this method does not estimate coefficients appropriately at one time. It will be a topic for future research. Furthermore we will focus on the theoretical investigation for the functional regression models with the sparse regularization. 


\section{References}

Araki, Y., Konishi, S., Kawano, S., and Matsui, H. (2009a), "Functional logistic discrimination via regularized basis expansions," Communications in Statistics-Theory and Methods, 38, 2944-2957.

- (2009b), "Functional regression modeling via regularized Gaussian basis expansions," Annals of the Institute of Statistical Mathematics, 61, 811-833.

Fan, J. and Li, R. (2001), "Variable selection via nonconcave penalized likelihood and its oracle properties," Journal of the American Statistical Association, 96, 1348-1360.

Ferraty, F. (2011), Recent Advances in Functional Data Analysis and Related Topics, Physica Verlag.

Ferraty, F. and Vieu, P. (2006), Nonparametric functional data analysis: theory and practice, Springer Verlag.

Friedman, J., Hastie, T., and Tibshirani, R. (2010), "Regularization paths for generalized linear models via coordinate descent," Journal of statistical software, 33, 1.

Hastie, T., Tibshirani, R., and Friedman, J. (2009), The elements of statistical learning 2nd ed., vol. 1, Springer Series in Statistics.

James, G. (2002), "Generalized linear models with functional predictors," Journal of the Royal Statistical Society: Series B, 64, 411-432.

James, G., Hastie, T., and Sugar, C. (2000), "Principal component models for sparse functional data," Biometrika, 87, 587-602.

Kawano, S. and Konishi, S. (2007), "Nonlinear regression modeling via regularized Gaussian basis functions," Bulletin of informatics and cybernetics, 39, 83-96.

Konishi, S., Ando, T., and Imoto, S. (2004), "Bayesian information criteria and smoothing parameter selection in radial basis function networks," Biometrika, 91, 27-43.

Krishnapuram, B., Carin, L., Figueiredo, M., and Hartemink, A. (2005), "Sparse multinomial logistic regression: Fast algorithms and generalization bounds," Pattern Analysis and Machine Intelligence, IEEE Transactions on, 27, 957-968.

Matsui, H. and Konishi, S. (2011), "Variable selection for functional regression models via the L1 regularization," Computational Statistics \& Data Analysis, 55, 3304-3310.

Obozinski, G., Taskar, B., and Jordan, M. (2010), "Joint covariate selection and joint subspace selection for multiple classification problems," Statistics and Computing, 20, $231-252$. 
Obozinski, G., Wainwright, M., and Jordan, M. (2011), "Support union recovery in highdimensional multivariate regression," The Annals of Statistics, 39, 1-47.

Park, M. and Hastie, T. (2007), "L1-regularization path algorithm for generalized linear models," Journal of the Royal Statistical Society: Series B, 69, 659-677.

Ramsay, J. and Silverman, B. (2002), Applied functional data analysis: methods and case studies, vol. 77, Springer, New York.

- (2005), Functional data analysis, Wiley Online Library.

Schwarz, G. (1978), "Estimating the dimension of a model," The annals of statistics, 6, 461-464.

Spellman, P., Sherlock, G., Zhang, M., Iyer, V., Anders, K., Eisen, M., Brown, P., Botstein, D., and Futcher, B. (1998), "Comprehensive identification of cell cycle-regulated genes of the yeast Saccharomyces cerevisiae by microarray hybridization," Molecular biology of the cell, 9, 3273-3297.

Tibshirani, R. (1996), "Regression shrinkage and selection via the lasso," Journal of the Royal Statistical Society. Series B (Methodological), 58, 267-288.

Turlach, B., Venables, W., and Wright, S. (2005), "Simultaneous variable selection," Technometrics, 47, 349-363.

Wang, H., Li, R., and Tsai, C. (2007), "Tuning parameter selectors for the smoothly clipped absolute deviation method," Biometrika, 94, 553-568.

Yuan, M., Ekici, A., Lu, Z., and Monteiro, R. (2007), "Dimension reduction and coefficient estimation in multivariate linear regression," Journal of the Royal Statistical Society: Series B, 69, 329-346.

Yuan, M. and Lin, Y. (2006), "Model selection and estimation in regression with grouped variables," Journal of the Royal Statistical Society: Series B, 68, 49-67.

Zou, H., Hastie, T., and Tibshirani, R. (2007), "On the "degrees of freedom" of the lasso," The Annals of Statistics, 35, 2173-2192. 
Table 1: Numbers of selected variables. Top: $P_{(1)}$, bottom: $P_{(2)}$.

\begin{tabular}{cc|cccc|cccc}
\hline \hline$P_{(1)}$ & $\sigma$ & \multicolumn{5}{|c|}{0.05} & \multicolumn{4}{c}{0.2} \\
\hline$n$ & $j$ & $\beta_{1 j} \beta_{2 j}$ & $\beta_{1 j}$ & $\beta_{2 j}$ & $\phi$ & $\beta_{1 j} \beta_{2 j}$ & $\beta_{1 j}$ & $\beta_{2 j}$ & $\phi$ \\
\hline 75 & 1 & 100 & 0 & 0 & 0 & 100 & 0 & 0 & 0 \\
& 2 & 37 & 0 & 63 & 0 & 33 & 0 & 67 & 0 \\
& 3 & 0 & 100 & 0 & 0 & 1 & 99 & 0 & 0 \\
& 4 & 0 & 25 & 6 & 69 & 12 & 45 & 16 & 27 \\
\hline 150 & 1 & 100 & 0 & 0 & 0 & 100 & 0 & 0 & 0 \\
& 2 & 32 & 0 & 68 & 0 & 49 & 0 & 51 & 0 \\
& 3 & 0 & 100 & 0 & 0 & 1 & 99 & 0 & 0 \\
& 4 & 1 & 33 & 2 & 64 & 15 & 42 & 14 & 29 \\
\hline 300 & 1 & 100 & 0 & 0 & 0 & 100 & 0 & 0 & 0 \\
& 2 & 45 & 0 & 55 & 0 & 34 & 0 & 66 & 0 \\
& 3 & 0 & 100 & 0 & 0 & 1 & 99 & 0 & 0 \\
& 4 & 2 & 32 & 5 & 61 & 26 & 40 & 15 & 19 \\
\hline
\end{tabular}

\begin{tabular}{cc|cccc|cccc}
\hline \hline$P_{(2)}$ & $\sigma$ & \multicolumn{5}{|c|}{0.05} & \multicolumn{4}{c}{0.2} \\
\hline$n$ & $j$ & $\beta_{1 j} \beta_{j 2}$ & $\beta_{1 j}$ & $\beta_{2 j}$ & $\phi$ & $\beta_{1 j} \beta_{2 j}$ & $\beta_{1 j}$ & $\beta_{2 j}$ & $\phi$ \\
\hline 75 & 1 & 100 & 0 & 0 & 0 & 100 & 0 & 0 & 0 \\
& 2 & 98 & 0 & 0 & 2 & 91 & 0 & 0 & 9 \\
& 3 & 100 & 0 & 0 & 0 & 99 & 0 & 0 & 1 \\
& 4 & 18 & 0 & 0 & 82 & 54 & 0 & 0 & 46 \\
\hline 150 & 1 & 100 & 0 & 0 & 0 & 100 & 0 & 0 & 0 \\
& 2 & 100 & 0 & 0 & 0 & 100 & 0 & 0 & 0 \\
& 3 & 100 & 0 & 0 & 0 & 100 & 0 & 0 & 0 \\
& 4 & 22 & 0 & 0 & 78 & 51 & 0 & 0 & 49 \\
\hline 300 & 1 & 100 & 0 & 0 & 0 & 100 & 0 & 0 & 0 \\
& 2 & 100 & 0 & 0 & 0 & 100 & 0 & 0 & 0 \\
& 3 & 100 & 0 & 0 & 0 & 100 & 0 & 0 & 0 \\
& 4 & 26 & 0 & 0 & 74 & 71 & 0 & 0 & 29 \\
\hline
\end{tabular}


Table 2: Averages of 100 training, test errors (\%) and the regularization parameter $\lambda$ (and its standard deviations in parenthesis). The top is for $P_{(1)}$ and the bottom is for $P_{(2)}$.

\begin{tabular}{c|ccc|ccc}
\hline \hline$\sigma$ & \multicolumn{3}{|c|}{0.05} & \multicolumn{3}{c}{0.2} \\
\hline$n$ & Train & Test & $\lambda \times 10^{3}$ & Train & Test & $\lambda \times 10^{3}$ \\
\hline 75 & 0.04 & 0.41 & $5.81(2.74)$ & 0.15 & 1.45 & $9.64(4.74)$ \\
150 & 0.10 & 0.33 & $3.79(2.02)$ & 0.35 & 1.12 & $6.51(3.20)$ \\
300 & 0.10 & 0.28 & $2.22(1.13)$ & 0.28 & 0.92 & $3.68(1.64)$ \\
\hline
\end{tabular}

\begin{tabular}{c|ccc|ccc}
\hline \hline$\sigma$ & \multicolumn{3}{|c|}{0.05} & \multicolumn{3}{c}{0.2} \\
\hline$n$ & Train & Test & $\lambda \times 10^{3}$ & Train & Test & $\lambda \times 10^{3}$ \\
\hline 75 & 0.08 & 0.80 & $6.28(2.94)$ & 0.27 & 1.93 & $12.69(6.28)$ \\
150 & 0.15 & 0.45 & $4.04(2.20)$ & 0.49 & 1.56 & $7.96(3.77)$ \\
300 & 0.12 & 0.38 & $2.37(1.27)$ & 0.40 & 1.17 & $4.29(1.58)$ \\
\hline
\end{tabular}

Table 3: Numbers of selected variables for $P_{(1)}$. Signs $i / j$ below indicate classification boundaries between $i$ and $j$ for $i, j=1, \ldots, 5$ with 1:G1, 2:G2/M, 3:M/G1, 4:S, 5:S/G2.

\begin{tabular}{c|cccccc}
\hline \hline & cln3 & clb2 & $\alpha$ & cdc15 & cdc28 & elu \\
\hline $1 / 2$ & 100 & 100 & 100 & 100 & 100 & 98 \\
$1 / 3$ & 100 & 100 & 100 & 99 & 100 & 100 \\
$1 / 4$ & 94 & 99 & 100 & 100 & 100 & 100 \\
$1 / 5$ & 100 & 100 & 100 & 100 & 100 & 100 \\
$2 / 3$ & 100 & 76 & 100 & 100 & 100 & 100 \\
$2 / 4$ & 100 & 100 & 99 & 100 & 100 & 100 \\
$2 / 5$ & 100 & 100 & 100 & 100 & 100 & 100 \\
$3 / 4$ & 100 & 47 & 100 & 100 & 100 & 100 \\
$3 / 5$ & 100 & 16 & 100 & 100 & 100 & 100 \\
$4 / 5$ & 100 & 82 & 100 & 100 & 98 & 98 \\
\hline
\end{tabular}




\section{List of MI Preprint Series, Kyushu University \\ The Global COE Program \\ Math-for-Industry Education \& Research Hub}

MI

MI2008-1 Takahiro ITO, Shuichi INOKUCHI \& Yoshihiro MIZOGUCHI

Abstract collision systems simulated by cellular automata

MI2008-2 Eiji ONODERA

The intial value problem for a third-order dispersive flow into compact almost Hermitian manifolds

MI2008-3 Hiroaki KIDO

On isosceles sets in the 4-dimensional Euclidean space

MI2008-4 Hirofumi NOTSU

Numerical computations of cavity flow problems by a pressure stabilized characteristiccurve finite element scheme

MI2008-5 Yoshiyasu OZEKI

Torsion points of abelian varieties with values in nfinite extensions over a p-adic field

MI2008-6 Yoshiyuki TOMIYAMA

Lifting Galois representations over arbitrary number fields

MI2008-7 Takehiro HIROTSU \& Setsuo TANIGUCHI

The random walk model revisited

MI2008-8 Silvia GANDY, Masaaki KANNO, Hirokazu ANAI \& Kazuhiro YOKOYAMA

Optimizing a particular real root of a polynomial by a special cylindrical algebraic decomposition

MI2008-9 Kazufumi KIMOTO, Sho MATSUMOTO \& Masato WAKAYAMA

Alpha-determinant cyclic modules and Jacobi polynomials

MI2008-10 Sangyeol LEE \& Hiroki MASUDA

Jarque-Bera Normality Test for the Driving Lévy Process of a Discretely Observed Univariate SDE

MI2008-11 Hiroyuki CHIHARA \& Eiji ONODERA

A third order dispersive flow for closed curves into almost Hermitian manifolds

MI2008-12 Takehiko KINOSHITA, Kouji HASHIMOTO and Mitsuhiro T. NAKAO

On the $L^{2}$ a priori error estimates to the finite element solution of elliptic problems with singular adjoint operator

MI2008-13 Jacques FARAUT and Masato WAKAYAMA

Hermitian symmetric spaces of tube type and multivariate Meixner-Pollaczek polynomials 
MI2008-14 Takashi NAKAMURA

Riemann zeta-values, Euler polynomials and the best constant of Sobolev inequality

MI2008-15 Takashi NAKAMURA

Some topics related to Hurwitz-Lerch zeta functions

MI2009-1 Yasuhide FUKUMOTO

Global time evolution of viscous vortex rings

MI2009-2 Hidetoshi MATSUI \& Sadanori KONISHI

Regularized functional regression modeling for functional response and predictors

MI2009-3 Hidetoshi MATSUI \& Sadanori KONISHI

Variable selection for functional regression model via the $L_{1}$ regularization

MI2009-4 Shuichi KAWANO \& Sadanori KONISHI

Nonlinear logistic discrimination via regularized Gaussian basis expansions

MI2009-5 Toshiro HIRANOUCHI \& Yuichiro TAGUCHII

Flat modules and Groebner bases over truncated discrete valuation rings

MI2009-6 Kenji KAJIWARA \& Yasuhiro OHTA

Bilinearization and Casorati determinant solutions to non-autonomous $1+1$ dimensional discrete soliton equations

MI2009-7 Yoshiyuki KAGEI

Asymptotic behavior of solutions of the compressible Navier-Stokes equation around the plane Couette flow

MI2009-8 Shohei TATEISHI, Hidetoshi MATSUI \& Sadanori KONISHI

Nonlinear regression modeling via the lasso-type regularization

MI2009-9 Takeshi TAKAISHI \& Masato KIMURA

Phase field model for mode III crack growth in two dimensional elasticity

MI2009-10 Shingo SAITO

Generalisation of Mack's formula for claims reserving with arbitrary exponents for the variance assumption

MI2009-11 Kenji KAJIWARA, Masanobu KANEKO, Atsushi NOBE \& Teruhisa TSUDA Ultradiscretization of a solvable two-dimensional chaotic map associated with the Hesse cubic curve

MI2009-12 Tetsu MASUDA

Hypergeometric T -functions of the q-Painlevé system of type $E_{8}^{(1)}$

MI2009-13 Hidenao IWANE, Hitoshi YANAMI, Hirokazu ANAI \& Kazuhiro YOKOYAMA A Practical Implementation of a Symbolic-Numeric Cylindrical Algebraic Decomposition for Quantifier Elimination

MI2009-14 Yasunori MAEKAWA

On Gaussian decay estimates of solutions to some linear elliptic equations and its applications 
MI2009-15 Yuya ISHIHARA \& Yoshiyuki KAGEI

Large time behavior of the semigroup on $L^{p}$ spaces associated with the linearized compressible Navier-Stokes equation in a cylindrical domain

MI2009-16 Chikashi ARITA, Atsuo KUNIBA, Kazumitsu SAKAI \& Tsuyoshi SAWABE

Spectrum in multi-species asymmetric simple exclusion process on a ring

MI2009-17 Masato WAKAYAMA \& Keitaro YAMAMOTO

Non-linear algebraic differential equations satisfied by certain family of elliptic functions

MI2009-18 Me Me NAING \& Yasuhide FUKUMOTO

Local Instability of an Elliptical Flow Subjected to a Coriolis Force

MI2009-19 Mitsunori KAYANO \& Sadanori KONISHI

Sparse functional principal component analysis via regularized basis expansions and its application

MI2009-20 Shuichi KAWANO \& Sadanori KONISHI

Semi-supervised logistic discrimination via regularized Gaussian basis expansions

MI2009-21 Hiroshi YOSHIDA, Yoshihiro MIWA \& Masanobu KANEKO

Elliptic curves and Fibonacci numbers arising from Lindenmayer system with symbolic computations

MI2009-22 Eiji ONODERA

A remark on the global existence of a third order dispersive flow into locally Hermitian symmetric spaces

MI2009-23 Stjepan LUGOMER \& Yasuhide FUKUMOTO

Generation of ribbons, helicoids and complex scherk surface in laser-matter Interactions

MI2009-24 Yu KAWAKAMI

Recent progress in value distribution of the hyperbolic Gauss map

MI2009-25 Takehiko KINOSHITA \& Mitsuhiro T. NAKAO

On very accurate enclosure of the optimal constant in the a priori error estimates for $H_{0}^{2}$-projection

MI2009-26 Manabu YOSHIDA

Ramification of local fields and Fontaine's property (Pm)

MI2009-27 Yu KAWAKAMI

Value distribution of the hyperbolic Gauss maps for flat fronts in hyperbolic threespace

MI2009-28 Masahisa TABATA

Numerical simulation of fluid movement in an hourglass by an energy-stable finite element scheme

MI2009-29 Yoshiyuki KAGEI \& Yasunori MAEKAWA

Asymptotic behaviors of solutions to evolution equations in the presence of translation and scaling invariance 
MI2009-30 Yoshiyuki KAGEI \& Yasunori MAEKAWA

On asymptotic behaviors of solutions to parabolic systems modelling chemotaxis

MI2009-31 Masato WAKAYAMA \& Yoshinori YAMASAKI

Hecke's zeros and higher depth determinants

MI2009-32 Olivier PIRONNEAU \& Masahisa TABATA

Stability and convergence of a Galerkin-characteristics finite element scheme of lumped mass type

MI2009-33 Chikashi ARITA

Queueing process with excluded-volume effect

MI2009-34 Kenji KAJIWARA, Nobutaka NAKAZONO \& Teruhisa TSUDA

Projective reduction of the discrete Painlevé system of type $\left(A_{2}+A_{1}\right)^{(1)}$

MI2009-35 Yosuke MIZUYAMA, Takamasa SHINDE, Masahisa TABATA \& Daisuke TAGAMI Finite element computation for scattering problems of micro-hologram using DtN map

MI2009-36 Reiichiro KAWAI \& Hiroki MASUDA

Exact simulation of finite variation tempered stable Ornstein-Uhlenbeck processes

MI2009-37 Hiroki MASUDA

On statistical aspects in calibrating a geometric skewed stable asset price model

MI2010-1 Hiroki MASUDA

Approximate self-weighted LAD estimation of discretely observed ergodic OrnsteinUhlenbeck processes

MI2010-2 Reiichiro KAWAI \& Hiroki MASUDA

Infinite variation tempered stable Ornstein-Uhlenbeck processes with discrete observations

MI2010-3 Kei HIROSE, Shuichi KAWANO, Daisuke MIIKE \& Sadanori KONISHI

Hyper-parameter selection in Bayesian structural equation models

MI2010-4 Nobuyuki IKEDA \& Setsuo TANIGUCHI

The Itô-Nisio theorem, quadratic Wiener functionals, and 1-solitons

MI2010-5 Shohei TATEISHI \& Sadanori KONISHI

Nonlinear regression modeling and detecting change point via the relevance vector machine

MI2010-6 Shuichi KAWANO, Toshihiro MISUMI \& Sadanori KONISHI

Semi-supervised logistic discrimination via graph-based regularization

MI2010-7 Teruhisa TSUDA

UC hierarchy and monodromy preserving deformation

MI2010-8 Takahiro ITO

Abstract collision systems on groups 
MI2010-9 Hiroshi YOSHIDA, Kinji KIMURA, Naoki YOSHIDA, Junko TANAKA \& Yoshihiro MIWA

An algebraic approach to underdetermined experiments

MI2010-10 Kei HIROSE \& Sadanori KONISHI

Variable selection via the grouped weighted lasso for factor analysis models

MI2010-11 Katsusuke NABESHIMA \& Hiroshi YOSHIDA

Derivation of specific conditions with Comprehensive Groebner Systems

MI2010-12 Yoshiyuki KAGEI, Yu NAGAFUCHI \& Takeshi SUDOU

Decay estimates on solutions of the linearized compressible Navier-Stokes equation around a Poiseuille type flow

MI2010-13 Reiichiro KAWAI \& Hiroki MASUDA

On simulation of tempered stable random variates

MI2010-14 Yoshiyasu OZEKI

Non-existence of certain Galois representations with a uniform tame inertia weight

MI2010-15 Me Me NAING \& Yasuhide FUKUMOTO

Local Instability of a Rotating Flow Driven by Precession of Arbitrary Frequency

MI2010-16 Yu KAWAKAMI \& Daisuke NAKAJO

The value distribution of the Gauss map of improper affine spheres

MI2010-17 Kazunori YASUTAKE

On the classification of rank 2 almost Fano bundles on projective space

MI2010-18 Toshimitsu TAKAESU

Scaling limits for the system of semi-relativistic particles coupled to a scalar bose field

MI2010-19 Reiichiro KAWAI \& Hiroki MASUDA

Local asymptotic normality for normal inverse Gaussian Lévy processes with highfrequency sampling

MI2010-20 Yasuhide FUKUMOTO, Makoto HIROTA \& Youichi MIE

Lagrangian approach to weakly nonlinear stability of an elliptical flow

MI2010-21 Hiroki MASUDA

Approximate quadratic estimating function for discretely observed Lévy driven SDEs with application to a noise normality test

MI2010-22 Toshimitsu TAKAESU

A Generalized Scaling Limit and its Application to the Semi-Relativistic Particles System Coupled to a Bose Field with Removing Ultraviolet Cutoffs

MI2010-23 Takahiro ITO, Mitsuhiko FUJIO, Shuichi INOKUCHI \& Yoshihiro MIZOGUCHI Composition, union and division of cellular automata on groups

MI2010-24 Toshimitsu TAKAESU

A Hardy's Uncertainty Principle Lemma in Weak Commutation Relations of HeisenbergLie Algebra 
MI2010-25 Toshimitsu TAKAESU

On the Essential Self-Adjointness of Anti-Commutative Operators

MI2010-26 Reiichiro KAWAI \& Hiroki MASUDA

On the local asymptotic behavior of the likelihood function for Meixner Lévy processes under high-frequency sampling

MI2010-27 Chikashi ARITA \& Daichi YANAGISAWA

Exclusive Queueing Process with Discrete Time

MI2010-28 Jun-ichi INOGUCHI, Kenji KAJIWARA, Nozomu MATSUURA \& Yasuhiro OHTA Motion and Bäcklund transformations of discrete plane curves

MI2010-29 Takanori YASUDA, Masaya YASUDA, Takeshi SHIMOYAMA \& Jun KOGURE On the Number of the Pairing-friendly Curves

MI2010-30 Chikashi ARITA \& Kohei MOTEGI

Spin-spin correlation functions of the $q$-VBS state of an integer spin model

MI2010-31 Shohei TATEISHI \& Sadanori KONISHI

Nonlinear regression modeling and spike detection via Gaussian basis expansions

MI2010-32 Nobutaka NAKAZONO

Hypergeometric $\tau$ functions of the $q$-Painlevé systems of type $\left(A_{2}+A_{1}\right)^{(1)}$

MI2010-33 Yoshiyuki KAGEI

Global existence of solutions to the compressible Navier-Stokes equation around parallel flows

MI2010-34 Nobushige KUROKAWA, Masato WAKAYAMA \& Yoshinori YAMASAKI

Milnor-Selberg zeta functions and zeta regularizations

MI2010-35 Kissani PERERA \& Yoshihiro MIZOGUCHI

Laplacian energy of directed graphs and minimizing maximum outdegree algorithms

MI2010-36 Takanori YASUDA

CAP representations of inner forms of $S p(4)$ with respect to Klingen parabolic subgroup

MI2010-37 Chikashi ARITA \& Andreas SCHADSCHNEIDER

Dynamical analysis of the exclusive queueing process

MI2011-1 Yasuhide FUKUMOTO\& Alexander B. SAMOKHIN

Singular electromagnetic modes in an anisotropic medium

MI2011-2 Hiroki KONDO, Shingo SAITO \& Setsuo TANIGUCHI

Asymptotic tail dependence of the normal copula

MI2011-3 Takehiro HIROTSU, Hiroki KONDO, Shingo SAITO, Takuya SATO, Tatsushi TANAKA \& Setsuo TANIGUCHI

Anderson-Darling test and the Malliavin calculus

MI2011-4 Hiroshi INOUE, Shohei TATEISHI \& Sadanori KONISHI

Nonlinear regression modeling via Compressed Sensing 
MI2011-5 Hiroshi INOUE

Implications in Compressed Sensing and the Restricted Isometry Property

MI2011-6 Daeju KIM \& Sadanori KONISHI

Predictive information criterion for nonlinear regression model based on basis expansion methods

MI2011-7 Shohei TATEISHI, Chiaki KINJYO \& Sadanori KONISHI

Group variable selection via relevance vector machine

MI2011-8 Jan BREZINA \& Yoshiyuki KAGEI

Decay properties of solutions to the linearized compressible Navier-Stokes equation around time-periodic parallel flow

Group variable selection via relevance vector machine

MI2011-9 Chikashi ARITA, Arvind AYYER, Kirone MALLICK \& Sylvain PROLHAC

Recursive structures in the multispecies TASEP

MI2011-10 Kazunori YASUTAKE

On projective space bundle with nef normalized tautological line bundle

MI2011-11 Hisashi ANDO, Mike HAY, Kenji KAJIWARA \& Tetsu MASUDA

An explicit formula for the discrete power function associated with circle patterns of Schramm type

MI2011-12 Yoshiyuki KAGEI

Asymptotic behavior of solutions to the compressible Navier-Stokes equation around a parallel flow

MI2011-13 Vladimír CHALUPECKÝ \& Adrian MUNTEAN

Semi-discrete finite difference multiscale scheme for a concrete corrosion model: approximation estimates and convergence

MI2011-14 Jun-ichi INOGUCHI, Kenji KAJIWARA, Nozomu MATSUURA \& Yasuhiro OHTA Explicit solutions to the semi-discrete modified KdV equation and motion of discrete plane curves

MI2011-15 Hiroshi INOUE

A generalization of restricted isometry property and applications to compressed sensing

MI2011-16 Yu KAWAKAMI

A ramification theorem for the ratio of canonical forms of flat surfaces in hyperbolic three-space

MI2011-17 Naoyuki KAMIYAMA

Matroid intersection with priority constraints

MI2012-1 Kazufumi KIMOTO \& Masato WAKAYAMA

Spectrum of non-commutative harmonic oscillators and residual modular forms

MI2012-2 Hiroki MASUDA

Mighty convergence of the Gaussian quasi-likelihood random fields for ergodic Levy driven SDE observed at high frequency 
MI2012-3 Hiroshi INOUE

A Weak RIP of theory of compressed sensing and LASSO

MI2012-4 Yasuhide FUKUMOTO \& Youich MIE

Hamiltonian bifurcation theory for a rotating flow subject to elliptic straining field

MI2012-5 Yu KAWAKAMI

On the maximal number of exceptional values of Gauss maps for various classes of surfaces

MI2012-6 Marcio GAMEIRO, Yasuaki HIRAOKA, Shunsuke IZUMI, Miroslav KRAMAR, Konstantin MISCHAIKOW \& Vidit NANDA

Topological Measurement of Protein Compressibility via Persistence Diagrams

MI2012-7 Nobutaka NAKAZONO \& Seiji NISHIOKA

Solutions to a $q$-analog of Painlevé III equation of type $D_{7}^{(1)}$

MI2012-8 Naoyuki KAMIYAMA

A new approach to the Pareto stable matching problem

MI2012-9 Jan BREZINA \& Yoshiyuki KAGEI

Spectral properties of the linearized compressible Navier-Stokes equation around time-periodic parallel flow

MI2012-10 Jan BREZINA

Asymptotic behavior of solutions to the compressible Navier-Stokes equation around a time-periodic parallel flow

MI2012-11 Daeju KIM, Shuichi KAWANO \& Yoshiyuki NINOMIYA

Adaptive basis expansion via the extended fused lasso

MI2012-12 Masato WAKAYAMA

On simplicity of the lowest eigenvalue of non-commutative harmonic oscillators

MI2012-13 Masatoshi OKITA

On the convergence rates for the compressible

Navier- Stokes equations with potential force

MI2013-1 Abuduwaili PAERHATI \& Yasuhide FUKUMOTO

A Counter-example to Thomson-Tait-Chetayev's Theorem

MI2013-2 Yasuhide FUKUMOTO \& Hirofumi SAKUMA

A unified view of topological invariants of barotropic and baroclinic fluids and their application to formal stability analysis of three-dimensional ideal gas flows

MI2013-3 Hiroki MASUDA

Asymptotics for functionals of self-normalized residuals of discretely observed stochastic processes

MI2013-4 Naoyuki KAMIYAMA

On Counting Output Patterns of Logic Circuits

MI2013-5 Hiroshi INOUE

RIPless Theory for Compressed Sensing 
MI2013-6 Hiroshi INOUE

Improved bounds on Restricted isometry for compressed sensing

MI2013-7 Hidetoshi MATSUI

Variable and boundary selection for functional data via multiclass logistic regression modeling 